\title{
NUMERICAL SIMULATION OF THE 3-D FLOW IN A CYLINDRICAL DUCT WITH TWO DIAPHRAGMS AT LOW MACH NUMBERS
}

\author{
YAROSLAV TROTSENKO ${ }^{1 *}$, IGOR VOVK ${ }^{2}$ \\ ${ }^{1}$ Taras Shevchenko National University of Kyiv, 03680 Kyiv, Ukraine \\ ${ }^{2}$ Institute of Hydromechanics, National Academy of Sciences of Ukraine, \\ 03057 Kyiv, Ukraine
}

[Received: 03 September 2019. Accepted: 30 April 2020]

doi: 10.7546/JTAM.50.20.02.08

\begin{abstract}
The three-dimensional flow of a viscous incompressible fluid in a cylindrical duct with two serial diaphragms is studied by the numerical solution of the non-stationary Navier-Stokes equations. The solution algorithm is based on the finite volume method using second-order accurate in both space and time difference schemes. It is shown that under certain conditions a sequential series of ring vortices is formed in the shear layer between the diaphragms. This causes self-oscillations of the pressure field in the neighbourhood of the second diaphragm orifice, as well as pressure fluctuations in the whole medium between the baffles. The flow structure has an azimuthal asymmetry that influences local features of the flow, but practically does not affect its integral characteristics.
\end{abstract}

KEY WORDS: 3-D flow, DNS, duct with baffles, vortical structures, selfsustained oscillations.

\section{INTRODUCTION}

Liquid (or gaseous) flows in irregular ducts are widely encountered in technical or scientific devices, as well as in nature: blood vessels, bronchial trees, gas distribution systems, corrugated pipes, solid propellant rocket motors etc. The structure of such flows is non-stationary due to the complex geometry of these systems. Under certain conditions this leads to self-sustained oscillations of the medium and, in consequence, the appearance of sound [1].

The emergence of a tone sound when a jet flows into a circular hole in a plate (hole-tone) was first observed by Sondhauss [2]. Then Rayleigh, based on his observations, proposed a feedback mechanism, according to which the jet cannot pass freely through the hole and, as a result, creates perturbations that are further transmitted upstream and give rise to new perturbations (Rayleigh bird-call) [3]. Later, Chanaud and Powell conducted a thorough study of the hole-tone phenomenon and

\footnotetext{
${ }^{*}$ Corresponding author e-mail: yaroslav.p.trotsenko@ gmail.com
} 
found that acoustic oscillations are associated with the periodic formation of vortex rings and their interaction with the plate [4]. Since then, intensive experimental studies of flows in ducts with baffles or cavities have been conducted (for example, [5-8]).

With the development of computer technology at the end of the 20th century, numerical simulations of such systems have become widespread. In the case of low Mach numbers, hybrid methods are preferred, according to which the general problem is decomposed into acoustic and hydrodynamic parts that are solved separately. This approach is used because the values of the fields and the lengths scales of these two problems vary considerably [9]. As a result, the corresponding methods differ in accuracy and requirements to computer resources.

For numerical simulations of flows in irregular ducts, two-dimensional vortex methods are initially used $[10,11]$. Today, the techniques of direct numerical simulation (DNS) and large eddy simulation (LES) are also actively used [12-15]. Due to advances in computer technology, it is also possible to numerically solve such problems in a three-dimensional formulation. For example, a three-dimensional incompressible discrete vortex method was used for studying the hole-tone phenomenon in [16]. In [17] the finite difference lattice Boltzmann method (FDLBM) was used for numerical analysis of the flow in the main steam stop valve (MSV) in thermal or nuclear power plants. In [18] the hole-tone phenomenon was studied by solving the unsteady three-dimensional fully compressible Navier-Stokes equations.

The aim of this work was to investigate the properties of the three-dimensional fluid flow in a cylindrical duct with two serial diaphragms. The solution was carried out by the DNS technique and was based on the main theses outlined in [19]. The proposed algorithm has previously been used to solve various problems of hydrodynamic acoustics and showed good agreement with experimental data and numerical results of other authors [20-22].

\section{Problem Formulation}

Consider the fluid flow in a semi-infinite cylindrical duct with two serial diaphragms. The diaphragms are identical and axisymmetric. In the longitudinal section of the duct depicted in Fig. 1, the diaphragms have semicircle contours near the holes. It was assumed that the surfaces of the duct and the baffles are immobile and absolutely rigid. The fluid flow with the density $\rho$ enters the computational region with the uniform velocity $V_{1}$ through the left boundary $(x=0)$ and leaves it through the right boundary $(x=L)$. The flow rate is considerably lower than the speed of sound in the medium $c$.

For calculations, the values of the geometric parameters of the diaphragms and the cavity between them were chosen such as in the experiments performed in [5]: $D_{2}=6.35 \mathrm{~mm}, D_{1} / D_{2}=D_{3} / D_{2}=8.16, L_{2} / D_{2}=1, L_{3} / D_{2}=6$. The length 


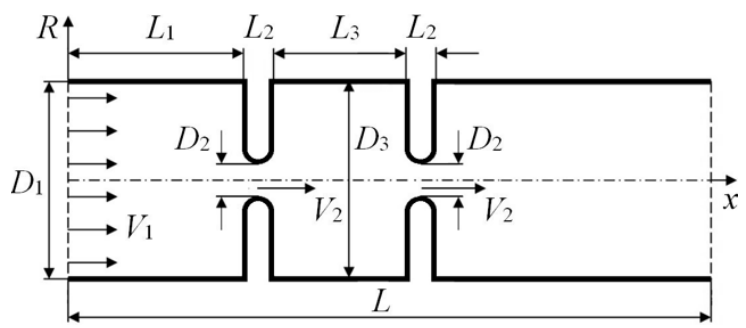

Fig. 1: Longitudinal section of the duct.

of the area upstream of the first diaphragm was set to $L_{1} / D_{2} \approx 7.87$, and the total length of the computational region was taken to be $L / D_{2} \approx 63.12$. The kinematic viscosity of the medium $\nu=1.5 \times 10^{-5} \mathrm{~m}^{2} / \mathrm{s}$ corresponds to the viscosity of the air at the temperature of $20^{\circ} \mathrm{C}$. The speed of sound in the medium is $c=343 \mathrm{~m} / \mathrm{s}$.

It is known that the sources of sound generated by the flow are small compared with the length of the sound wave [5]. Therefore, the following hypotheses were accepted: the power of the acoustic oscillations excited by the flow is much less than the power of the stream itself and the generated sound does not affect the character of the flow. Due to this, the problem was solved within the framework of the model of a viscous incompressible fluid.

The main parameter of the problem is the Reynolds number, which can be defined as $R e=V_{2} D_{2} / \nu$, where $V_{2}$ is the flow rate averaged over the diaphragm orifice. In this case, the scale of the length is the diameter of the aperture $D_{2}$, the velocity scale is the speed $V_{2}$, the time scale is specified as $D_{2} / V_{2}$, and the pressure scale is defined as the double dynamic pressure $\rho V_{2}^{2}$.

Within the framework of the accepted model, the process is described by the system of the non-stationary Navier-Stokes equations, which in dimensionless quantities in a vector form can be written as

$$
\begin{aligned}
& \frac{\partial \mathbf{U}}{\partial t}+(\mathbf{U} \cdot \nabla) \mathbf{U}=\frac{1}{R e} \Delta \mathbf{U}-\nabla p, \\
& \nabla \cdot \mathbf{U}=0,
\end{aligned}
$$

where $p$ is the pressure scalar field, and $\mathbf{U}$ is the velocity vector field.

The following boundary conditions for the velocity were set: a uniform flow at the entry to the computational region, the condition of sticking on the solid surfaces $\Sigma$ and the "soft" boundary condition corresponding to the equality of the normal velocity gradient to zero at the exit from the computational region

$$
\left.\mathbf{U}\right|_{x=0}=\left(V_{1}, 0,0\right),\left.\quad \mathbf{U}\right|_{\Sigma}=\mathbf{0},\left.\quad \frac{\partial \mathbf{U}}{\partial x}\right|_{x=L}=\mathbf{0} .
$$


The research was conducted for four input speed values $V_{1} \in\{0.08 ; 0.1 ; 0.12$; $0.14\} \mathrm{m} / \mathrm{s}$, which corresponds to the Reynolds numbers $R e \in\{2255 ; 2819 ; 3383$; $3946\}$. Thus, the Mach number, which can be defined as $M=V_{2} / c$, did not exceed the value of $M \approx 0.027$.

For pressure, the condition of equality of its normal gradient to zero was set on the entire boundary of the computational region, except the exit from the region, where the constant pressure equal to zero was chosen

$$
\left.\frac{\partial p}{\partial x}\right|_{x=0}=0,\left.\quad \frac{\partial p}{\partial \mathbf{n}}\right|_{\Sigma}=0,\left.\quad p\right|_{x=L}=0 .
$$

At the initial moment for the first calculation, the medium was motionless:

$$
\mathbf{U}(t=0)=\mathbf{0}, \quad p(t=0)=0 .
$$

In the following calculations, under the initial conditions, the pressure and velocity fields obtained in the previous calculation, corresponding to the formed motion after the end of the transient processes were set:

$$
\mathbf{U}(t=0)=\mathbf{U}_{\text {pre }}, \quad p(t=0)=p_{\text {pre }} .
$$

\section{Numerical Algorithm}

The solution of the set problem was carried out numerically using the finite volume method. In the calculations, the toolbox libraries with OpenFOAM public code were used. The computational grid was generated with a help of a freely supplied finiteelement mesh generator equipped with the GMSH public code. A non-orthogonal block structured grid with concentration of nodes near the diaphragms and in their orifices was used. Figures 2 (a) and (b) show the discretization of the solid surfaces in the area of the diaphragms and the computational grid in the neighbourhood of the second aperture in half the longitudinal section of the duct, respectively. The number of control volumes in the radial direction of the diaphragm orifice is 35 with the smallest step of $8 \cdot 10^{-5} \mathrm{~m}$ near the surface; the number of control volumes in the azimuthal direction is 72 with the step of $5^{\circ}$; and the total number of cells is approximately $N \approx 2.7 \times 10^{6}$.

To find the integrals over the control volumes, the generalized Gauss procedure was used. The normal velocity gradients on the surfaces of the control volumes required for the determination of the diffusion terms by the Gauss theorem were computed according to the velocity values in the centroids of the adjacent volumes by using a second-order scheme. In the non-orthogonal sections of the grid, an iteration procedure of correction of the errors caused by the non-orthogonality of the grid was applied [23]. 


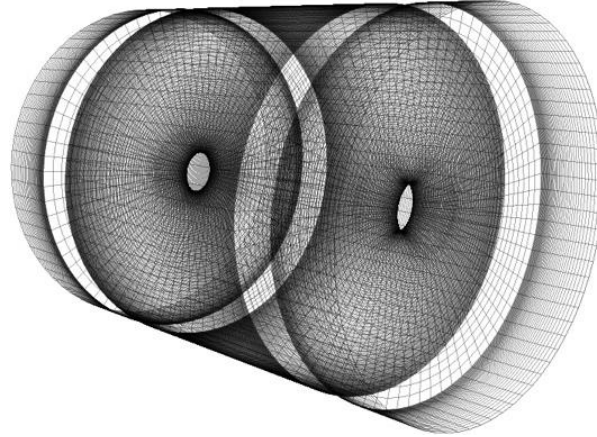

(a)

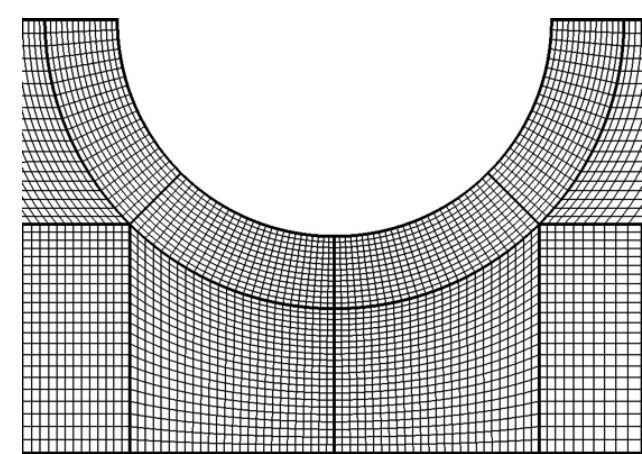

(b)

Fig. 2: Computational region: (a) solid surfaces in the area of the diaphragms; (b) half the longitudinal section in the neighbourhood of the second aperture.

For the interpolation of the convective terms, the TVD (total-variation diminishing) form of a central-difference scheme for a vector field with a Sweby-type fluxlimiter was used:

$$
\mathbf{U}_{F}=\left(\mathbf{U}_{F}\right)_{\mathrm{UD}}+\Psi\left[\left(\mathbf{U}_{F}\right)_{\mathrm{CD}}-\left(\mathbf{U}_{F}\right)_{\mathrm{UD}}\right],
$$

where $\left(\mathbf{U}_{F}\right)_{\mathrm{UD}}$ is the velocity value on the face of the control volume calculated according to the first-order upwind difference scheme, $\left(\mathbf{U}_{F}\right)_{\mathrm{CD}}$ is the velocity value on the cell face calculated according to the second-order central difference scheme. $\Psi$ is a non-linear flux-limiter defined as a function of the ratio of velocity gradients at the faces between the control volume and its neighbours upstream and downstream

$$
\begin{aligned}
& \Psi(q)=\max \left(\min \left(\frac{2}{k} q, 1\right), 0\right), \\
& q=2 \frac{\left(\mathbf{U}_{\mathrm{M}}-\mathbf{U}_{\mathrm{K}}\right)\left(\mathbf{U}_{\mathrm{N}}-\mathbf{U}_{\mathrm{M}}\right)}{\left(\mathbf{U}_{\mathrm{N}}-\mathbf{U}_{\mathrm{M}}\right)^{2}}-1,
\end{aligned}
$$

where $\mathrm{K}, \mathrm{M}, \mathrm{N}$ are the three centroids sequentially arranged in the direction of the flow [24]. In this paper, the coefficient $k=1$ is chosen, which corresponds to the best stability of the calculation.

An implicit three-point asymmetric second-order scheme with backward differences was chosen as the scheme of the time derivative discretization. For the combined evaluation of the velocity and pressure fields, the PISO (pressure implicit split operator) procedure was used [25]. For the solution of the constructed system of linearized algebraic equations, PCG and PBiCG (preconditioned (bi-)conjugate gradient) iterative solvers were used for symmetric and asymmetric matrices, respectively. 
As a preconditioner, DIC (diagonal-based incomplete Cholesky preconditioner) and DILU (diagonal-based incomplete LU preconditioner based on a simplified incomplete LU factorization) were chosen for the symmetric and asymmetric matrices, respectively [26]. A more detailed description of the applied numerical procedure can be found in [19]. HPC computing resources was provided by the supercomputer SCIT of Institute of Cybernetics NAS of Ukraine [27].

In order to control the accuracy of the calculations, the velocity field was numerically integrated over the cross sections of the apertures $S_{1}\left(x=L_{1}+L_{2} / 2\right)$ and $S_{2}$ $\left(x=L_{1}+3 L_{2} / 2+L_{3}\right)$. This controls the condition of medium incompressibility, which can be represented in the form

$$
\left|\frac{4 \int_{S_{i}} U_{x} d s-\pi D_{1}^{2} V_{1}}{\pi D_{1}^{2} V_{1}}\right|<\delta, \quad i=1,2 .
$$

For the results presented in this paper, the relative error $\delta$ did not exceed the value of $8 \times 10^{-3}$.

In order to identify vortex structures in the three-dimensional flow, the Q-method was used in this paper. According to this method a vortex is defined as a region in which the second invariant $Q$ of the tensor $\nabla \mathbf{U}$ is positive

$$
Q=\frac{1}{2}\left((\operatorname{tr}(\nabla \mathbf{U}))^{2}-\operatorname{tr}\left((\nabla \mathbf{U})^{2}\right)\right)=\frac{1}{2}\left(\|\boldsymbol{\Omega}\|^{2}-\|\mathbf{S}\|^{2}\right)>0 .
$$

Here $\mathbf{S}$ and $\boldsymbol{\Omega}$ are the symmetric and the antisymmetric parts of the tensor $\nabla \mathbf{U}$, respectively; $\|\boldsymbol{\Omega}\|$ and $\|\mathbf{S}\|$ are the norms of these tensors: $\|\boldsymbol{\Omega}\|^{2}=\operatorname{tr}\left(\boldsymbol{\Omega} \cdot \boldsymbol{\Omega}^{T}\right)$, $\|\mathbf{S}\|^{2}=\operatorname{tr}\left(\mathbf{S} \cdot \mathbf{S}^{T}\right)$ [28]. The first member of the right-hand side of (11) is responsible for the rotation of an elementary volume, and the second term is responsible for its stretching/compression. Thus, in addition to the local rotation in the region, the Q-method also requires that the rotation dominate the stretching.

\section{RESUlts AND ANALYSIS}

Two-dimensional model of an axisymmetric flow in the considered duct was investigated in [29]. It was shown that in the range of Reynolds numbers $R e \in[2678 ; 4228]$ the fluid flow in the region between the diaphragms was non-stationary and was characterized by the presence of an unstable shear layer. The ring vortices formed in the shear layer impinged on the second diaphragm, and caused oscillations of the velocity and pressure fields near the aperture. These oscillations were quasiperiodic and made it possible to estimate the frequency of the excited sound.

Consider now the results obtained within the model of a three-dimensional flow. Figure 3 shows iso-surfaces of the invariant $Q$ of the tensor $\nabla \mathbf{U}$ for the four values of 
the Reynolds number. The iso-surfaces are coloured in the accordance with the magnitude of the dimensional flow velocity $U^{*}$. At $R e=2255$, ring vortices are formed downstream of the second diaphragm, which convect downstream and collapse at a certain distance from the diaphragm (Fig. 3 (a)). These vortices are almost axisymmetric. However, vortices do not occur in the region between the diaphragms. That is, the shear layer becomes stationary after the completion of the transient processes. Therefore, the tone sound generated by the interaction of the vortices with the solid surface of the diaphragm is not excited.

With the increase of the Reynolds number to $R e=2819$, the ring vortices are formed in the shear layer when approaching the second diaphragm. Figure 3 (b) clearly shows that they are asymmetric. Tilt from the duct axis and azimuthal deformation of the vortices are observed. These vortices impinge on the second diaphragm and cause quasiperiodic oscillations of the velocity and pressure fields in the vicinity of its orifice. In addition, there is a simultaneous fluctuation of the pressure of the whole medium between the diaphragms. The periods of these oscillations coincide and have the value of $T \approx 1.83 \times 10^{-3} \mathrm{~s}$ in this case, which matches with the oscillation period obtained for the axisymmetric model $T_{S} \approx 1.83 \times 10^{-3} \mathrm{~s}$ [29].

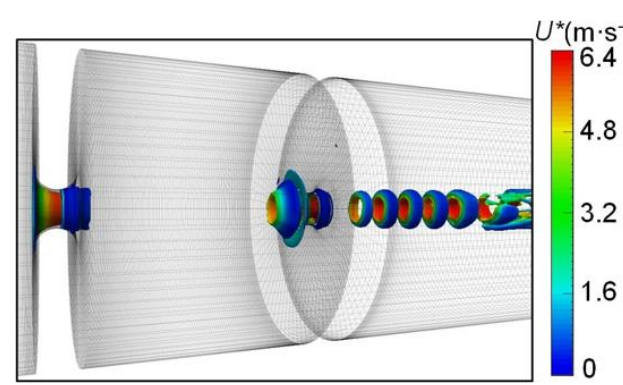

(a)

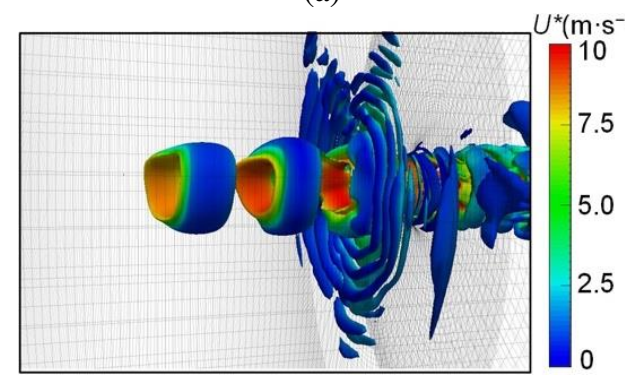

(c)

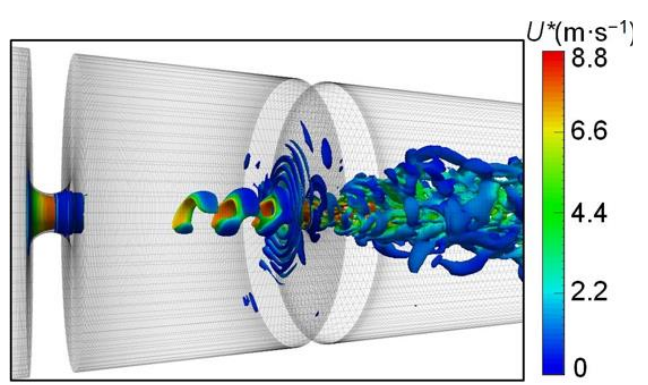

(b)

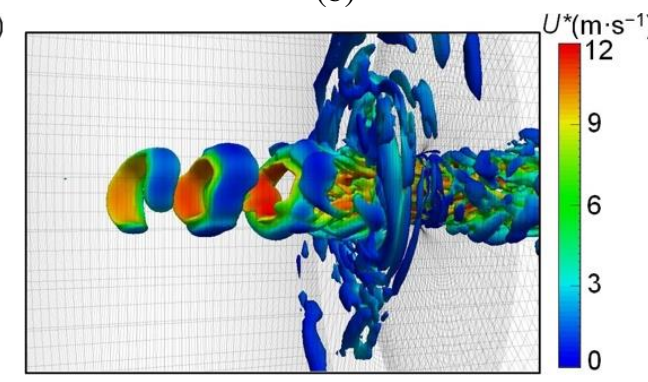

(d)

Fig. 3: Iso-surfaces of the second invariant $Q$ of the velocity gradient tensor $\nabla \mathbf{U}$ : (a) $R e=2255, Q=0.016$; (b) $R e=2819, Q=0.025$; (c) $R e=3383, Q=0.054$; (d) $R e=3946, Q=0.117$. 
Consequently, in spite of the difference between the local features of the axisymmetric and the three-dimensional flows, the integral characteristics of the medium self-oscillations coincide.

Figures 3 (c) and 3 (d) show the iso-surfaces $Q$ in the neighbourhood of the second aperture. With the increase of the Reynolds number to $R e=3383$, the flow structure remains similar to the previous case. Figure 3 (c) clearly shows as after the collision of vortices with the diaphragm, their parts spread radially into the cavity. In this case, the oscillation period is $T \approx 1.53 \times 10^{-3} \mathrm{~s}$, which is also consistent with the period obtained for the axisymmetric model $T_{S} \approx 1.52 \times 10^{-3} \mathrm{~s}$. At $R e=3946$, the flow in the region between the diaphragms is turbulent. The formation of ring vortices in the shear layer is preserved, but they begin to collapse as they approach the second diaphragm (Fig. 3 (d)). As a result, the formation of the ring vortices in the shear layer loses its periodicity. In this case, the acoustic oscillations take a noise character. Quasiperiodic oscillations were still observed at this flow velocity in the case of the axisymmetric model. This result is natural because the three-dimensional flow is less stable than the axisymmetric flow.

Figure 4 shows dimensional pressure fields $p^{*}$ in the longitudinal section of the duct in the vicinity of the second aperture for four time moments $\tilde{t}$ during a one period

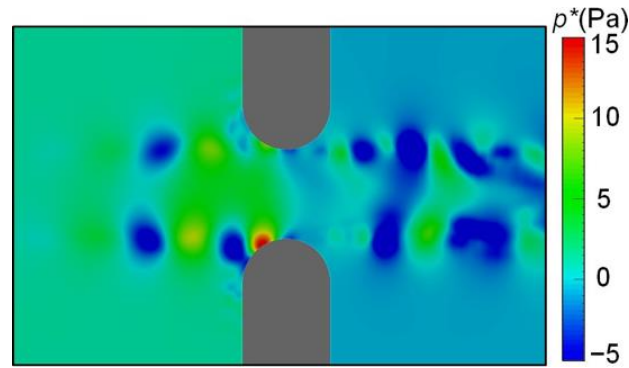

(a)

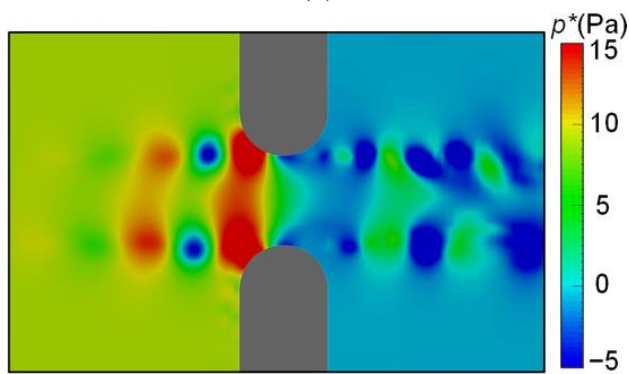

(c)

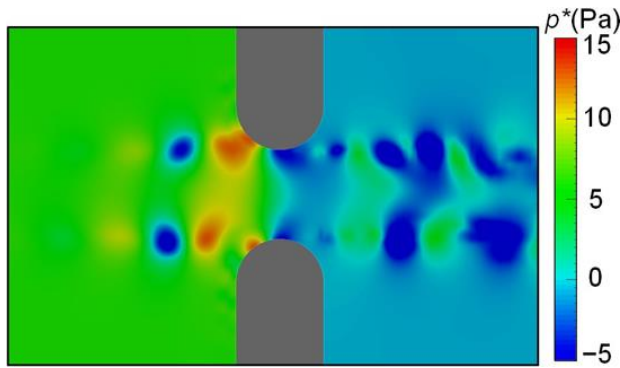

(b)

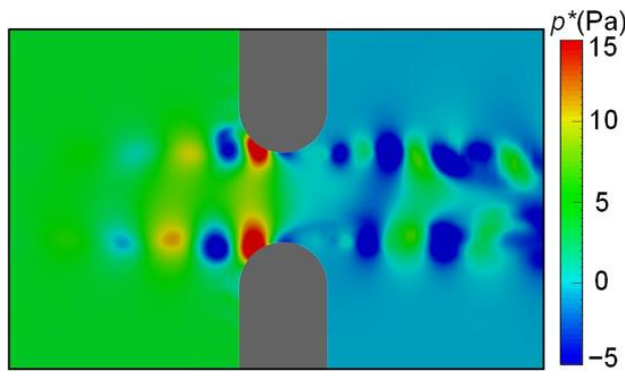

(d)

Fig. 4: Pressure fields $p^{*}$ in the longitudinal section of the duct in the vicinity of the second aperture at $R e=3383$ : (a) $\tilde{t}=0$; (b) $\tilde{t}=T / 4$; (c) $\tilde{t}=T / 2$; (d) $\tilde{t}=3 T / 4$. 
of oscillations $T$ at $R e=3383$. The regions of low pressure upstream from the second diaphragm correspond to the ring vortices. Between the vortices the regions of high pressure are observed. Figure 4 (a) shows the moment of the minimum total pressure in the cavity at the beginning of the oscillation period at $\tilde{t}=0$. Hence, when the vortex impinges on the diaphragm surface, the total pressure in the cavity is reduced. After the destruction of the vortex, when its part passes through the aperture, the pressure begins to increase (Fig. 4 (b)). When the next vortex approaches the diaphragm, a high pressure zone is established between the vortex and the diaphragm, resulting in an increase in the total pressure in the cavity. Figure 4 (c) shows the moment of the maximum total pressure at $\tilde{t}=T / 2$. When the vortex convects closer to the diaphragm, the high pressure zone decreases gradually and the total pressure falls (Fig. 4 (d)).

The obtained results are in good agreement with experimental researches and numerical simulations of other authors. In particular, an asymmetry of the flow in a hole-tone system with the absolute geometric axial symmetry was observed in [18]. The azimuthal deformation and slight tilting of ring vortices in the shear layer were noticed. However, the authors noted that the asymmetry was much less apparent in the pressure fluctuation fields, and therefore they neglected the effects of inhomogeneity in the azimuthal direction. At the same time, it was shown that the magnitude of the asymmetry depends on the geometric parameters of the system and develops with the increase in the flow velocity [7].

It should be noted that, the acoustic feedback, which may arise due to the excitation of standing acoustic waves in the duct both downstream and upstream of the diaphragms, is not investigated in this work. It is known that such a resonance can determine the characteristics of self-sustained oscillations $[6,12]$. In this regard, the obtained self-oscillation frequency at $R e=3383$ is higher than the sound frequency $f_{\exp } \approx 540 \mathrm{~Hz}$ in the experiment [5]. At the same time, at $R e=2819$, the frequencies of the oscillations are much closer to each other $\left(f_{\exp } \approx 525 \mathrm{~Hz}\right)$.

\section{CONCLUSIONS}

Numerical simulation of the three-dimensional flow of viscous incompressible fluid in a cylindrical duct with two diaphragms was carried out. The solution was based on the finite volume method using second-order accurate in both space and time difference schemes. For the interpolation of the convective terms, the TVD form of a central-difference scheme with a flux-limiter was used. The combined calculation of the velocity and pressure fields was carried out using the PISO procedure.

An analysis of the properties of the flow in the region between the diaphragms was carried out, depending on the flow velocity. It was shown that under certain conditions a series of ring vortices is formed in the shear layer that cause quasiperi- 
odic oscillations of the pressure field in the vicinity of the second aperture. These oscillations may serve as a source of sound in the duct. It was also shown that the three-dimensional flow is essentially asymmetric, despite the axisymmetry of the geometry of the system, as well as the boundary and initial conditions. However, the integral characteristics, such as the frequency of the formation of ring vortices in the shear layer and their impinging on the surface of the second diaphragm, as well as the fluctuation of the total pressure in the medium between the diaphragms, practically coincide with the model of an axisymmetric flow.

The results are in good agreement with the results of other authors. Calculated values of the velocity and pressure fields give an opportunity to estimate the frequency of excited acoustic oscillations and can be used to study the acoustic field.

\section{REFERENCES}

[1] I.V. Vovk, V.T. GRInchenko (2010) "The Flow-Generated Sound (Essay about an Aerohydrodynamical Acoustics)". Naukova Dumka, Kyiv (in Russian).

[2] C. Sondhauss (1854) Ueber die beim Ausströmen der Luft entstehenden Töne. Annalen der Physik 167(1) 126-147.

[3] J.W.S. RAYLEIGH (1945) "The Theory of Sound", Vol. 2. Dover publications, New York.

[4] R.C. Chanaud, A. Powell (1965) Some Experiments Concerning the Hole and Ring Tone. Journal of the Acoustical Society of America 37(5) 902-911.

[5] T.A. Wilson, G.S. Beavers, M.A. DeCoster, D.K. Holger, M.D. RegenFUSS (1971) Experiments on the Fluid Mechanics of Whistling. Journal of the Acoustical Society of America $\mathbf{5 0}$ 366-372.

[6] X.Y. Huang, D.S. Weaver (1991) On the Active Control of Shear Layer Oscillations across a Cavity in the Presence of Pipeline Acoustic Resonance, Journal of Fluids and Structures 5(2) 207-219.

[7] A.J. Meganathan, A.D. Vakili (2006) An Experimental Study of Acoustic and Flow Characteristics of Hole Tones. In: 44th AIAA Aerospace Sciences Meeting and Exhibit, Reno, Nevada, p. 1015.

[8] S. Mohamed, S. Ziada (2018) Particle Image Velocimetry Measurements of Aeroacoustic Sources of a Shallow Cavity. Journal of Pressure Vessel Technology 140041702.

[9] N. CuRle (1955) The Influence of Solid Boundaries upon Aerodynamic Sound. Proceedings of The Royal Society A Mathematical Physical and Engineering Sciences 231 505-514.

[10] K. Hourigan, M.C. Welsh, M.C. Thompson, A.N. Stokes (1990) Aerodynamic Sources of Acoustic Resonance in a Duct with Baffles. Journal of Fluids and Structures 4(4) 345-370.

[11] M.A. Langthjem, M. Nakano (2005) A Numerical Simulation of the Hole-Tone Feedback Cycle Based on an Axisymmetric Discrete Vortex Method and Curle's Equation. Journal of Sound and Vibration 288(1-2) 133-176. 
[12] G. Nakiboglu, H.B.M. Manders, A. Hirschberg (2012) Aeroacoustic Power Generated by a Compact Axisymmetric Cavity: Prediction of Self-Sustained Oscillation and Influence of the Depth. Journal of Fluid Mechanics 703 163-191.

[13] J. Golliard, N. Gonzalez-Diez, S. Belfroid, G. Nakiboglu, A. HiRschBERG (2013) U-RANS Model for the Prediction of the Acoustic Sound Power Generated in a Whistling Corrugated Pipe. In: Proceedings of ASME 2013 Pressure Vessels and Piping Conference, Paris, France, 4, p. 97385.

[14] B. Rajavel, M.G. Pras AD (2014) Parametric Studies on Acoustics of Corrugated Tubes using Large Eddy Simulation (LES). Noise Control Engineering Journa 62(4) 218-231.

[15] N. Khaoua, L. Khezzar, M. Alshehhi (2017) Flow Dynamics of a Plane Jet Impinging on a Slotted Plate: Large Eddy Simulation. Journal of Applied Fluid Mechanics 10(4) 1211-1222.

[16] M.A. Langthuem, M. Nakano (2015) A Numerical Study of the Hole-Tone Phenomenon Subjected to Non-Axisymmetric Shape Perturbations of the Jet Nozzle. Theoretical and Computational Fluid Dynamics 29(3) 127-153.

[17] A. TAmura, S. TAKahashi, S. Sato, S. Hori (2013) Numerical Analysis of Sound in the Main Steam Stop Valve, and a Sound Suppression Method. Journal of Fluid Science and Technology 8(1) 120-135.

[18] K. Matsuura, M. Nakano (2012) A Throttling Mechanism Sustaining a Hole Tone Feedback System at Very Low Mach Numbers. Journal of Fluid Mechanics 710569 605.

[19] V.S. Malyuga (2010) Numerical Investigation of the Flow in a Duct with Two Serial Stenoses. Algorithm of the Solution. Journal “Applied Hydromechanics” 12(4) 45-62. (in Russian)

[20] I.V. Vovk, V.S. Malyuga (2010) On One Method of Sound Field Evaluation for Aeolian Tones. Acoustic Bulletin 13(2) 3-19 (in Russian).

[21] I.V. Vovk, V.T. Grinchenko, V.S. Malyuga (2017) Self-Induced Oscillations of a Jet Flowing over the Wedge. The Mechanism of Appearance of the Feedback. Journal of Mathematical Sciences 220(3) 265-272.

[22] V.S. Malyuga (2013) Numerical Simulation of Viscous Incompressible Flow Past a Sphere. Journal “Applied Hydromechanics” 15(3) 43-67 (in Russian).

[23] H. JASAK (1996) Error Analysis and Estimation for the Finite Volume Method with Applications to Fluid Flows, PhD, Imperial College London.

[24] N.P. WAterson, H. Deconinck (2007) Design Principles for Bounded HigherOrder Convection Schemes - a Unified Approach. Journal of Computational Physics 224(1) 182-207.

[25] R.I. IsS A (1986) Solution of Implicitly Discretised Fluid Flow Equations by OperatorSplitting. Journal of Computational Physics 62(1) 40-65.

[26] R. Barrett, M. Berry, T.F. Chan, J. Demmel, J.M. Donato, J. Dongarra, V. Eijkhout, R. Pozo, C. Romine, H. VAn Der Vorst (1994) "Templates for the Solution of Linear Systems: Building Blocks for Iterative Methods", 2nd Edition. SIAM, Philadelphia. 
[27] A.L. Golovynskyi, A.L. Malenko, I.V. Sergienko, V.G. Tulchinsky (2013) Power Efficient Supercomputer SCIT-4. Bull. Nat. Acad. Sci. Ukraine 50-59 (in Ukrainian).

[28] J. JeOng, F. Huss ain (1995) On the Identification of a Vortex. Journal of Fluid Mechanics 285 69-94.

[29] I.V. Vovk, V.T. Matsypura, YA.P. Trotsenko (2019) Excitation of SelfSustained Oscillations by a Fluid Flow in a Cylindrical Duct with Two Diaphragms. Nonlinear Oscillations 22(1) 36-53 (in Ukrainian) 